\title{
Corrigendum to "Sum of dilates of two sets" [Notes on Number Theory and Discrete Mathematics, Vol. 23, 2017, No. 4, 34-41]
}

\author{
Raj Kumar Mistri \\ Department of Mathematics, Harish-Chandra Research Institute, HBNI \\ Chhatnag Road, Jhunsi, Allahabad - 211 019, India \\ e-mail: itsrajhans@gmail.com
}

It was discovered that the original online version of the paper "Sum of dilates of two sets" contained incomplete address of the institute of the author. The complete address appears above. The correction was already made in the online version, available at

http://nntdm. net/volume-23-2017/number-4/34-41/.

Unfortunately, the discovery of the error was too late to make correction in the printed issue. 